\title{
AMPK/TSC2/mTOR pathway regulates replicative senescence of human vascular smooth muscle cells
}

\author{
JUN-KUN ZHAN, YAN-JIAO WANG, SHUANG LI, YI WANG, PAN TAN, JE-YU HE, \\ YI-YIN CHEN, HUI-QIAN DENG, WU HUANG, XIAO LIN and YOU-SHUO LIU
}

\begin{abstract}
Geriatric Department, The Second Xiangya Hospital, Institute of Aging and Geriatrics, Central South University, Changsha, Hunan 410011, P.R. China
\end{abstract}

Received August 31, 2016; Accepted May 19, 2017

DOI: $10.3892 / \mathrm{etm} .2018 .6767$

\begin{abstract}
Age-associated diseases, including vascular diseases, are on the rise with the increase in the aging population. However, the mechanisms of aging and age-associated vascular dysfunction remain to be fully elucidated. Replicative senescence of vascular smooth muscle cells (VSMCs) contributes to aging as well as age-associated vascular diseases. Rapamycin may delay aging-associated diseases via inhibition of the mammalian target of rapamycin (mTOR), but its role in VSMC aging has remained elusive. The present study investigated the involvement of mTOR signaling in replicative senescence of VSMCs. Replicative senescence was induced in human VSMCs by extended passages and identified by assessing the cell morphology, senescence-associated $\beta$-galactosidase activity, and p53 and p21 protein expression. Protein expression and phosphorylation were determined by western blot analysis. Significant senescence of VSMCs was observed in cells subjected to extended passaging (until passage 15). Significant decreases in adenosine monophosphate-activated protein kinase (AMPK)/tuberous sclerosis complex 2 (TSC2) phosphorylation, but significant increases in $\mathrm{mTOR} /$ ribosomal protein S6 kinase 1 (S6K1) phosphorylation, were observed in cells with replicative senescence compared with those in young cells. Pre-treatment of VSMCs with AMPK activator and mTOR inhibitor delayed replicative senescence and reversed changes in AMPK $\alpha$, TSC2, mTOR and S6K1 phosphorylation in senescent VSMCs. The AMPK/TSC2/mTOR/S6K1 signaling axis was found to have an important role in regulating replicative senescence of human VSMCs.
\end{abstract}

Correspondence to: Dr You-Shuo Liu, Geriatric Department, The Second Xiangya Hospital, Institute of Aging and Geriatrics, Central South University, 139 Middle Renmin Road, Changsha, Hunan 410011, P.R. China

E-mail: liuyoushuo@yeah.net

Key words: vascular smooth muscle cells, replicative senescence, AMPK, TSC2, mTOR, vascular aging

\section{Introduction}

Age-associated vascular diseases are accompanied by structural and functional changes in blood vessels. A body of evidence suggested that the behavior of vascular smooth muscle cells (VSMCs) is modified with ageing (1), and senescent VSMCs have been observed in atherosclerotic plaques of patients with atherosclerosis (2). Thus, senescent VSMCs contribute to ageing and age-associated cardiovascular diseases. Although permanent inhibition of cell proliferation is thought to be a traditional hallmark of cellular senescence, this procedure is accompanied by changes in the expression of replicative senescence-associated genes, such as p53, p21 and senescence-associated $\beta$-galactosidase (SA- $\beta$-gal) (3).

Mammalian target of rapamycin (mTOR) is a serine/threonine protein kinase and its functions rely on forming two distinct complexes, mTOR complex 1 (mTORC1) and mTORC2 (4). Dysfunctional mTOR signaling has been considered a central integral mechanism linked to ageing (5). AMP-activated protein kinase (AMPK) is a highly conserved heterotrimeric kinase complex. Activation of AMPK leads to the inhibition of mTORC1 via a tuberous sclerosis complex 2 (TSC2)-dependent or -independent mechanism (6). In animal models with impaired mTORC1 signaling, AMPK is highly activated (7), suggesting a negative crosstalk between these two pathways. AMPK/TSC2/mTOR is a classic upstream pathway of mTORC1 signaling, which affects the phosphorylation of downstream factors, such as ribosome protein subunit 6 kinase 1 (S6K1), to regulate cell metabolism, proliferation, differentiation and autophagy. However, the role of mTOR and particularly that of its downstream effector S6K1 in age-associated vascular diseases has remained largely elusive. A previous study has demonstrated that inhibition of the mTORC1/S6K1 pathway by rapamycin or activation of AMPK by 5 -aminoimidazole-4-carboxamide riboside (AICAR) negatively regulates mTORC1 signaling and attenuated pressure overload-induced cardiac hypertrophy in vivo (8). Conversely, a deficiency in AMPK expression was reported to enhance mTOR/S6K1 signaling and exacerbate myocardial hypertrophy in response to pressure overload (9). However, the role of the AMPK/mTOR signaling in VSMC senescence has remained elusive.

The purpose of the present study was to explore the roles of AMPK/TSC2/mTOR signaling in the replicative senescence 
of VSMCs and to provide a theoretical basis for the clinical prevention and treatment of vascular aging.

\section{Materials and methods}

Cell culture. Human VMSCs were purchased from the American Type Culture Collection (ATCC; Manassas, VA, USA; ATCC ${ }^{\circledR}$ no. CRL-1999). Cells were cultured in Vascular Cell Basal Medium (ATCC). When cells reached 90\% confluence, $20 \%$ of the cells were re-plated and cultured until they reached $90 \%$ confluence again for the next passage and were then passaged every 3-4 days. The VSMC phenotype was confirmed by positive immunohistochemical staining for $\alpha$-smooth muscle actin using anti- $\alpha$-SMA antibody (catalogue no. 55135-1-AP; 1:100 dilution; Proteintech, Chicago, IL, USA) following the manufacture's protocol.

Cell treatments. Human VSMCs were divided into five treatment groups: i) Young cell group [5th passage (P5)], ii) replicative senescence group (P15), iii) replicative senescence + rapamycin group [10 nM rapamycin (Calbiochem Corp; EMD Millipore, Billerica, MA, USA) was added to the medium from P5-15], iv) replicative senescence + Compound C group [20 $\mu \mathrm{m} / 1 \mathrm{AMPK}$ inhibitor Compound C (Calbiochem Corp; EMD Millipore) was added from P5-15] and v) replicative senescence + AICAR group [0.1 mM AMPK activator AICAR (Calbiochem Corp; EMD Millipore) was added from P5-15].

Western blot analysis. Cells were lysed and the protein concentration was measured using a BCA Protein Assay kit (Beyotime Institute of Biotechnology, Haimen, China). Total protein (20 $\mu \mathrm{g}$ per lane) was separated by $12 \%$ SDS-PAGE and transferred onto nitrocellulose membranes (Pierce; Thermo Fisher Scientific, Inc., Waltham, MA, USA). After blocking with 5\% non-fat milk for $45 \mathrm{~min}$, membranes were incubated with primary antibodies overnight at $4{ }^{\circ} \mathrm{C}$, followed by incubation with secondary antibody Anti-mouse IgG, HRP-linked Antibody (catalogue no. 7076) or anti-rabbit IgG, HRP-linked antibody (catalogue no. 7074) both at a dilution of 1:2,000, purchased from Cell Signaling Technology, Inc. (Danvers, MA, USA) for $2 \mathrm{~h}$ at room temperature. Reactive proteins were visualized using chemiluminescent reagents (Pierce; Thermo Fisher Scientific, Inc., Waltham, MA, USA). As a loading control, the blots were probed with GAPDH antibody (catalogue no. SC-365062; Santa Cruz Biotechnology, Inc., Dallas, TX, USA). Primary antibodies for AMPKa, (catalogue no. 5831), p-mTOR Ser ${ }^{2481}$ (catalogue no. 2974), phospho (p)-AMPK $\alpha \mathrm{Thr}^{172}$ (catalogue no. 2535), mTOR (catalogue no. 2972), p-mTOR Ser ${ }^{2448}$ (catalogue no. 2971), TSC2 (catalogue no. 3612) and p-TSC2 (catalogue no. 3615) were purchased from Cell Signaling Technology, Inc. Primary antibodies for p-mTOR Thr ${ }^{2446}$ (catalogue no. ab63552), ALP (ab133602) S6K1 (catalogue no. ab32529), p-S6K1Thr ${ }^{389}$ (catalogue no. ab2571) and P21 (catalogue no. ab102635) were purchased from Abcam (Cambridge, MA, USA). Primary antibodies for osteocalcin (catalogue no. SC-74495) and p53 (catalogue no. SC-6243), were purchased from Santa Cruz Biotechnology, Inc.

SA- $\beta$-gal activity. SA- $\beta$-gal activity was measured according to a previously established protocol (10). SA- $\beta$-gal staining was performed using a senescence-galactosidase staining kit (Cell Signaling Technology, Inc., Danvers, MA, USA) by following the manufacturer's manual. After the blue staining became visible in either experimental or control cells, 500 cells in each treatment group were counted under a light microscope.

Statistical analysis. Values are expressed as the mean \pm standard deviation. Statistical analysis was performed using SPSS v17.0 (SPSS, Inc., Chicago, IL, USA). Differences between two groups were analyzed using the Student's t-test. $\mathrm{P}<0.05$ was considered to indicate a statistically significant difference.

\section{Results}

Inhibition of the AMPK/TSC2/mTOR pathway inhibits replicative senescence of VSMCs. Human VSMCs were treated as described in the Methods section. The percentage of SA- $\beta$-gal-positive cells was increased in the replicative senescence, replicative senescence + rapamycin, replicative senescence + Compound $\mathrm{C}$ and replicative senescence + AICAR groups compared with that of young cells $(\mathrm{P}<0.001$; Fig. 1A and B). The senescent cells presented as irregular or triangular shapes with obvious pseudopodia and larger overall size compared with that of normal cells. Intracellular vacuoles were seen in a proportion of the senescent cells. The percentage of SA- $\beta$-gal positive cells was decreased in the replicative senescence + rapamycin $(\mathrm{P}<0.001)$, replicative senescence + $\mathrm{CC}(\mathrm{P}<0.001)$ and replicative senescence + AICAR $(\mathrm{P}<0.001)$ groups compared with that in the replicative senescence group. The percentage of SA- $\beta$-gal-positive cells in the replicative senescence + Compound $\mathrm{C}$ group was significantly higher than that in the replicative senescence + rapamycin and replicative senescence + AICAR groups $(\mathrm{P}<0.05, \mathrm{P}<0.01$, respectively). The percentage of SA- $\beta$-gal positive cells in young cell group was significantly lower than that in all other groups $(\mathrm{P}<0.05$; Fig. 1A and B).

p53 and p21 are senescence-associated proteins. Western blot analysis demonstrated that p53 and p21 protein levels were significantly higher in cells with replicative senescence compared with those in young cells. However, in the rapamycin- and AICAR-treated senescent cells, p53 and p21 protein levels were significantly lowered $(\mathrm{P}<0.01)$ and were significantly lower than those in the Compound $\mathrm{C}$-treated senescent cells $(\mathrm{P}<0.01$; Fig. $1 \mathrm{C}$ and $\mathrm{D})$. These findings suggested that mTOR signaling was involved in the replicative senescence of VSMCs and that rapamycin and AICAR inhibits replicative senescence.

The AMPK/TSC2/mTOR signaling pathway regulates the replicative senescence of VSMCs. The mTOR protein expression and the levels of p-mTOR $\left(\mathrm{S}^{2448}\right)$, p-mTOR $\left(\mathrm{T}^{2446}\right)$ and p-S6K1 protein were significantly higher in the replicative senescence, replicative senescence + rapamycin, replicative senescence + Compound $\mathrm{C}$ and replicative senescence + AICAR groups compared with those in young cells (Fig. 2). The levels in the rapamycin- and AICAR- treated replicative senescence groups were significantly lower than those in the replicative senescence and Compound C-treated replicative senescence groups (Fig. 2). No significant differences in 

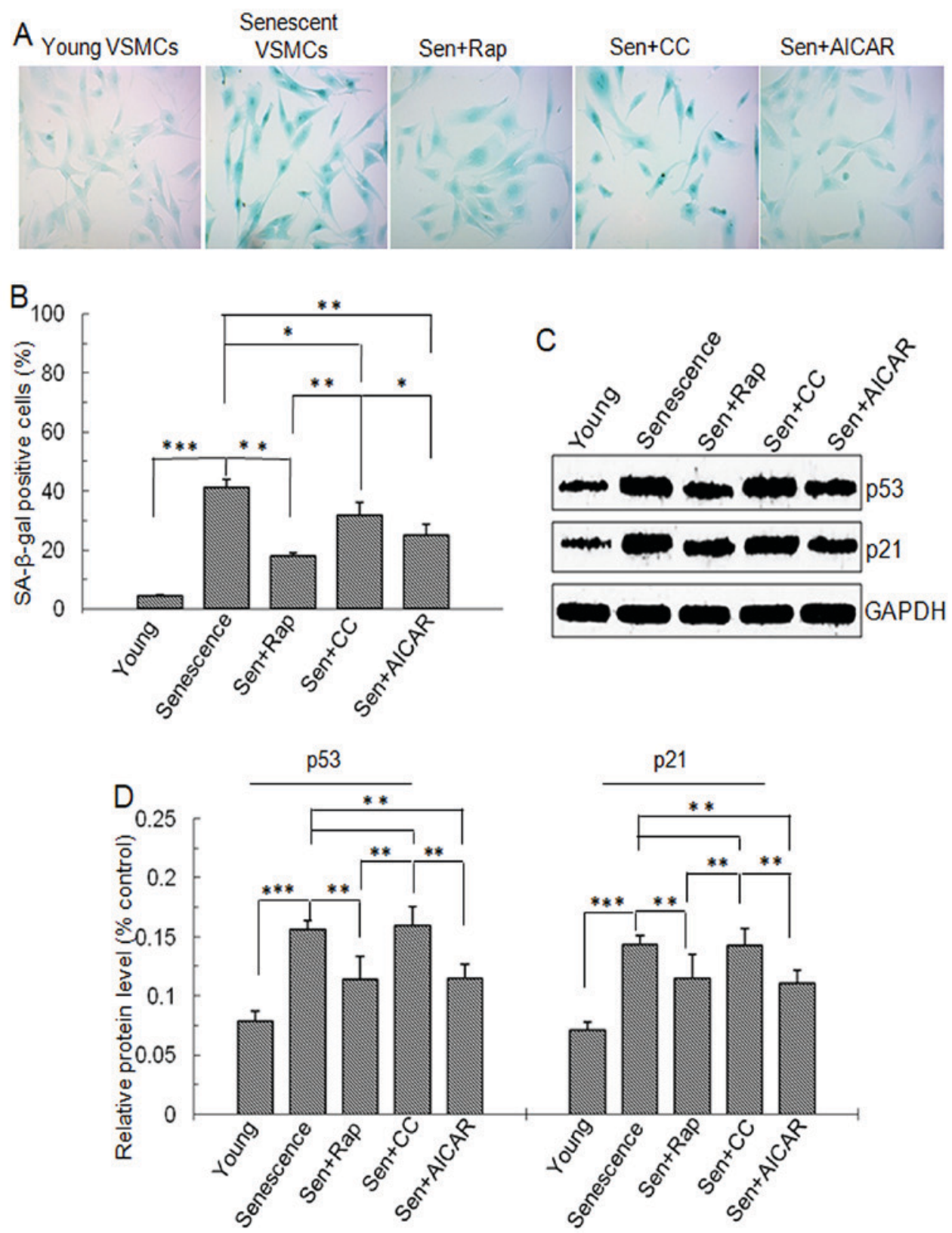

Figure 1. SA- $\beta$-gal staining and p53 and p21 protein level. Vascular smooth muscle cells were divided into five groups: Young cells, senescent cells, Sen + rap, Sen + CC and Sen + AICAR group. (A) SA- $\beta$-gal staining (blue). Magnification x200. (B) Quantification SA- $\beta$-gal-stained cells. (C) Representative western blots displaying p53 and p21 protein expression. (D) Semi-quantitative analysis of p53 and p21 protein levels in C. Values are expressed as the mean \pm standard deviation $(\mathrm{n}=4) .{ }^{*} \mathrm{P}<0.05,{ }^{* *} \mathrm{P}<0.01,{ }^{* * *} \mathrm{P}<0.001$ between two indicated groups. Sen, senescent cells; Rap, rapamycin; CC, Compound C; SA- $\beta$-gal, senescence-associated $\beta$-galactosidase, AICAR, 5-aminoimidazole-4-carboxamide riboside (agonist of adenosine monophosphate-activated protein kinase).

p-mTOR $\left(\mathrm{S}^{2481}\right)$ and S6K1 levels were observed between the 5 groups (Fig. 2).

To further investigate the upstream signaling of mTOR, AMPK and TSC2 in the treatment groups were examined by western blot analysis. The results demonstrated that $\mathrm{p}$-AMPK $\alpha$ $\mathrm{Thr}^{172}$ and p-TSC2 protein levels were significantly lower in the replicative senescence, replicative senescence + rapamycin, replicative senescence + Compound $\mathrm{C}$ and replicative senescence + AICAR groups compared with those in young cells (Fig. 3). p-AMPK $\alpha$ Thr $^{172}$ and p-TSC2 protein levels in the Compound $\mathrm{C}$-treated replicative senescence group were significantly lower than those in the AICAR- and rapamycin-treated replicative senescence groups (Fig. 3). p-AMPK $\alpha$ Thr $^{172}$ and p-TSC2 protein levels in the rapamycin- and AICAR-treated replicative senescence groups were significantly higher than those in the replicative senescence group. p-TSC2 protein levels in the Compound C-treated replicative senescence group were significantly lower than those in the replicative senescence group $(\mathrm{P}<0.05$; Fig. 3B).

\section{Discussion}

Calcification of VSMCs is widely observed in a variety of cardiovascular diseases. However, VSMC calcification has 

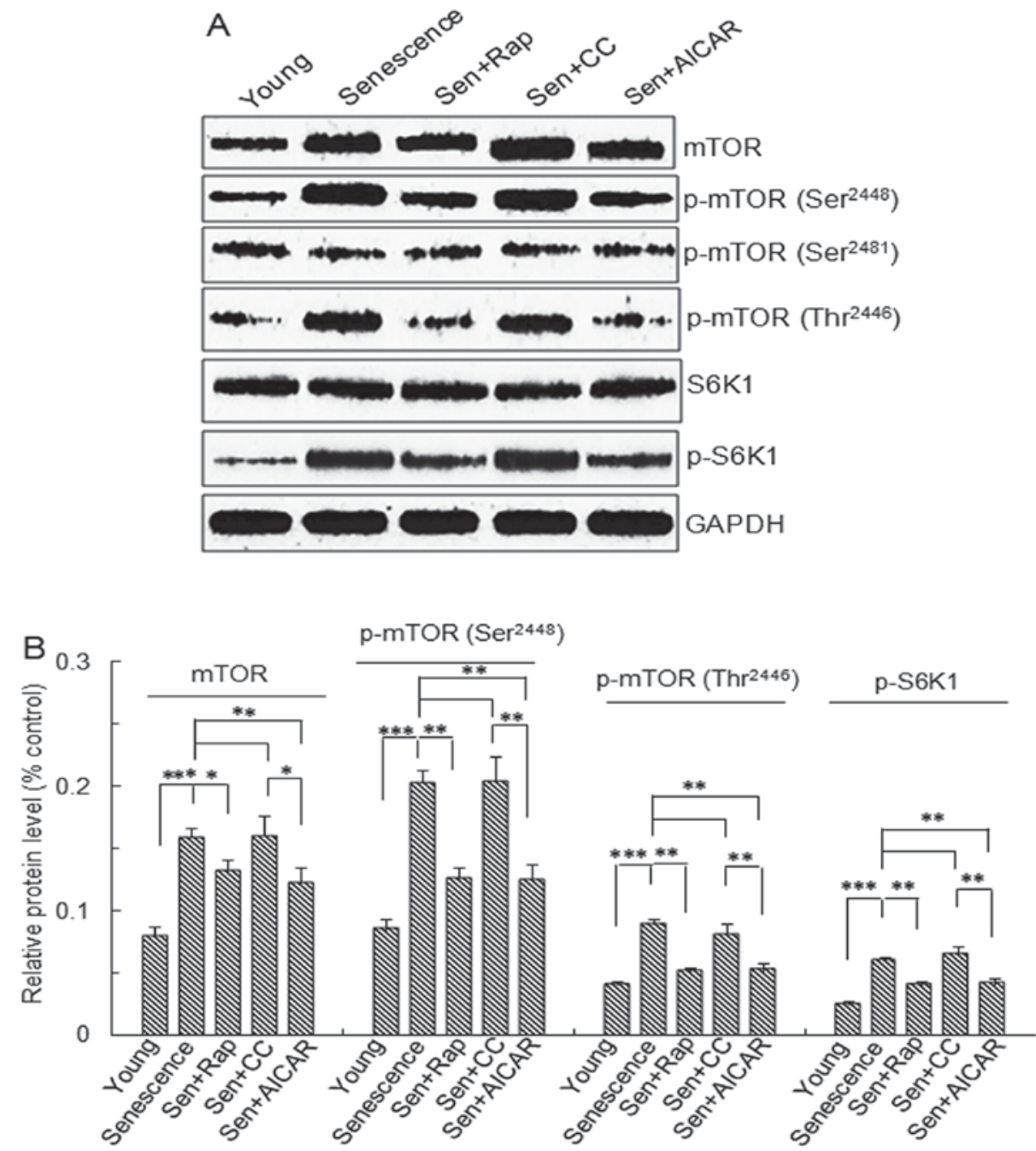

Figure 2. mTOR and S6K1 protein expression and phosphorylation. Vascular smooth muscle cells were divided into five groups: Young cells, senescent cells, Sen + rap, Sen + CC and Sen + AICAR group. (A) Representative western blots of mTOR, p-mTOR $\left(\mathrm{Ser}^{2448}\right)$, p-mTOR (Thr $\left.{ }^{2446}\right), \mathrm{p}-\mathrm{mTOR}\left(\mathrm{Ser}^{2481}\right), \mathrm{S} 6 \mathrm{~K} 1$ and p-S6K1 protein levels. (B) Semi-quantitative analysis of mTOR, p-mTOR $\left(\mathrm{Ser}^{2448}\right)$, p-mTOR $\left(\mathrm{Thr}^{2446}\right)$ and p-S6K1 protein levels in A. Values are expressed as the mean \pm standard deviation $(\mathrm{n}=4) .{ }^{*} \mathrm{P}<0.05,{ }^{* * *} \mathrm{P}<0.01,{ }^{* * *} \mathrm{P}<0.001$ between two indicated groups. Sen, senescent cells; Rap, rapamycin; CC, Compound $\mathrm{C}$; p-mTOR, phosphorylated mammalian target of rapamycin; S6K1, ribosomal protein S6 kinase 1; AICAR, 5-aminoimidazole-4-carboxamide riboside (agonist of adenosine monophosphate-activated protein kinase).

also been revealed to be a manifestation of cellular senescence. In other words, replicative senescence of VSMCs enhances age-associated medial artery calcification $(11,12)$. A previous study revealed that the AMPK-TSC2-mTOR-S6K1 signal pathway is involved in osteoblastic differentiation and calcification of VSMCs and this may be markedly inhibited following adiponectin treatment (13). Tan et al (14) demonstrated that inhibition of the PI3K/Akt/mTOR/S6K1 signal pathway decreased replicative senescence in human VSMCs. GLP-1 analogue liraglutide may also attenuate osteoblastic differentiation and calcification of human VSMCs through regulating PI3K/Akt/mTOR/S6K1 signaling (15). The present study demonstrated that AMPK/TSC2/mTOR/S6K1 signaling is involved in replicative senescence of VSMCs. In the present study, replicative senescence of VSMCs was induced in vitro by passaging for 15 generations. Significant increases in SA- $\beta$-gal activity, p53 and p 21 protein expression as well as increase in cell size were observed in VSMCs at 15 passages. Cell volume increases, changes in the expression of replicative senescence-associated genes, such as p53 and $\mathrm{p} 21$, and SA- $\beta$-gal activity are widely thought of as markers of cellular senescence (3). Thus, the replicative senescence model of VSMCs was successfully established by extended passaging. Application of rapamycin, an mTORC1 inhibitor, significantly inhibited the replicative senescence of VSMCs. Further molecular study revealed that the expression of mTOR, as well as the phosphorylation of mTOR at $\mathrm{Ser}^{2448}$ and $\mathrm{Thr}^{2446}$ was significantly increased in VSMCs with replicative senescence. Of note, rapamycin significantly inhibited mTOR expression and phosphorylation. The present findings suggested that the mTOR pathway has a role in VSMC senescence. Of note, to the best of our knowledge, the present study was the first to reveal that mTOR phosphorylation at $\mathrm{Ser}^{2448}$ at $\mathrm{Thr}^{2446}$, but not at $\mathrm{Ser}^{2481}$, regulates replicative senescence of VSMCs.

S6K1 is a specific downstream signaling protein and substrate of mTORC1, which regulates S6K1 activation through its phosphorylation at $\mathrm{Thr}^{389}$. In the present study, 

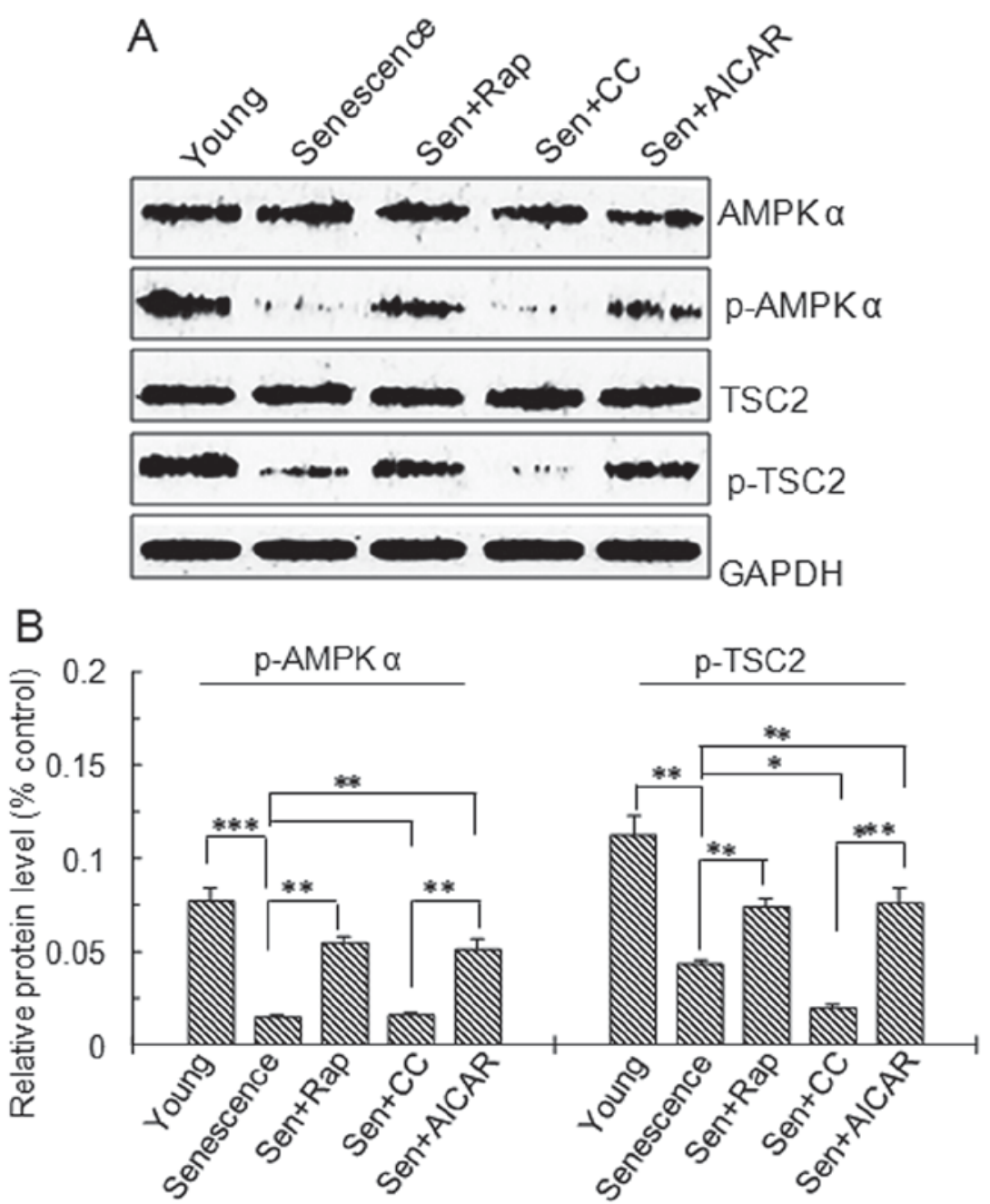

Figure 3. AMPK and TSC2 protein expression and phosphorylation. Vascular smooth muscle cells were divided into five groups: Young cells, senescent cells, Sen + rap, Sen + CC and Sen + AICAR group. (A) Representative western blots of AMPK $\alpha$, p-AMPK $\alpha$ (Thr ${ }^{172}$ ), TSC2 and p-TSC2 protein levels. (B) Semi-quantitative analysis of AMPK $\alpha, \mathrm{p}-\mathrm{AMPK} \alpha\left(\mathrm{Thr}^{172}\right), \mathrm{TSC} 2$ and $\mathrm{p}-\mathrm{TSC} 2$ protein levels in A. Values are expressed as the mean \pm standard deviation $(\mathrm{n}=4) .{ }^{*} \mathrm{P}<0.05,{ }^{* *} \mathrm{P}<0.01,{ }^{* * * *} \mathrm{P}<0.001$ between two indicated groups. Sen, senescent cells; Rap, rapamycin; CC, Compound C; p-AMPK, phosphorylated adenosine monophosphate-activated protein kinase; AICAR, 5-aminoimidazole-4-carboxamide riboside (agonist of adenosine monophosphate-activated protein kinase); TSC2, tuberous sclerosis complex 2.

AICAR and rapamycin applied during extended passaging inhibited mTOR signaling and S6K1 phosphorylation as well as senescence of VSMCs. These findings suggested that the $\mathrm{mTOR} / \mathrm{S} 6 \mathrm{~K} 1$ pathway is involved in the process of replicative senescence of VSMCs. A previous study demonstrated that the activation of AMPK leads to the inhibition of mTORC1 via a TSC2-dependent or -independent mechanism (6). To validate whether AMPK/TSC2 signaling occurs upstream of mTOR activation, AMPK and TSC2 protein expression and phosphorylation were determined. In senescent VSMCs, AMPK and TSC2 protein phosphorylation, but not their expression, was significantly decreased. Application of the AMPK activator AICAR significantly inhibited SA- $\beta$-gal activity as well as p53 and p21 protein expression, suggesting that AMPK activator inhibited replicative senescence in VSMCs. However, application of AMPK inhibitor Compound C did not significantly accelerate replicative senescence. This result may require further comparative observation using a small number of passages. Further molecular study identified that AMPK activator AICAR significantly increased TSC2 phosphorylation, while inhibiting mTOR expression and phosphorylation at $\mathrm{Ser}^{2448}$ and $\mathrm{Thr}^{2446}$ residues as well as S6K1 phosphorylation, while the effect of AMPK inhibitor Compound $\mathrm{C}$ on mTOR signing was not significant. These findings suggested that the downregulation of AMPK/TSC2 signaling and subsequent upregulation of mTOR/S6K1 signaling are involved in the senescence of VSMCs in vitro.

Of note, the mTOR inhibitor rapamycin significantly inhibited mTOR expression and phosphorylation accompanied by a significant increase in AMPK $\alpha$ and TSC2 phosphorylation. A previous study using an animal model demonstrated that AMPK is highly activated if mTORC1 signaling is impaired (16), suggesting that mTOR signaling exerts a feedback regulation on AMPK/TSC2/mTOR signaling. These findings suggested that mTOR signaling has an important role in the replicative senescence of VSMCs and that activation of the AMPK/TSC2/mTOR/S6K1 pathway may delay the replicative senescence of VSMCs. 
In conclusion, the AMPK/TSC2/mTOR/S6K1 signaling pathway has a key role in the replicative senescence of VSMCs. Interference with mTOR signaling may be used to inhibit replicative senescence of VSMCs.

\section{Acknowledgements}

The present study was supported by the National Basic Research Program of China (grant no. 2014CB910500), the National Natural Science Foundation of China (grant nos. 81501212, 81370931) and the Hunan Province Special Health Research Projects (grant no. A2015-04).

\section{Competing interests}

The authors declare that they have no competing interests.

\section{References}

1. Monk BA and George SJ: The effect of ageing on vascular smooth muscle cell behaviour-a mini-review. Gerontology 61: 416-426, 2015

2. Yin $\mathrm{H}$ and Pickering JG: Cellular senescence and vascular disease: Novel routes to better understanding and therapy. Can J Cardiol 32: 612-623, 2016.

3. Unterluggauer H, Hütter E, Voglauer R, Grillari J, Vöth M, Bereiter-Hahn J, Jansen-Dürr P and Jendrach M: Identification of cultivation-independent markers of human endothelial cell. senescence in vitro. Biogerontology 8: 383-397, 2007.

4. Hay N and Sonenberg N: Upstream and downstream of mTOR. Genes Dev 18: 1926-1945, 2004.

5. Zoncu R, Efeyan A and Sabatini DM: mTOR: From growth signal integration to cancer, diabetes and ageing. Nat Rev Mol Cell Biol 12: 21-35, 2011

6. Kawaguchi T, Hayakawa M, Koga $\mathrm{H}$ and Torimura T: Effects of fucoidan on proliferation, AMP-activated protein kinase, and downstream metabolism- and cell cycle-associated molecules in poorly differentiated human hepatoma HLF cells. Int J Oncol 46 : 2216-2222, 2015.
7. Long YC, Cheng Z, Copps KD and White MF: Insulin receptor substrates Irs1 and Irs2 coordinate skeletal muscle growth and metabolism via the Akt and AMPK pathways. Mol Cell Biol 31: 430-441, 2011.

8. Li HL, Yin R, Chen D, Liu D, Wang D, Yang Q and Dong YG Long-term activation of adenosine monophosphateactivated protein kinase attenuates pressure-overload-induced cardiac hypertrophy. J Cell Biochem 100: 1086-1099, 2007.

9. Zhang P, Hu X, Xu X, Fassett J, Zhu G, Viollet B, Xu W, Wiczer B, Bernlohr DA, Bache RJ and Chen Y: AMP activated protein kinase-alpha 2 deficiency exacerbates pressure-overload-induced left ventricular hypertrophy and dysfunction in mice. Hypertension 52: 918-924, 2008.

10. Dimri GP, Lee X, Basile G, Acosta M, Scott G, Roskelley C, Medrano EE, Linskens M, Rubelj I, Pereira-Smith O, et al: A biomarker that identifies senescent human cells in culture and in aging skin in vivo. Proc Natl Acad Sci USA 92: 9363-9367, 1995.

11. Nakano-Kurimoto R, Ikeda K, Uraoka M, Nakagawa Y, Yutaka K, Koide M, Takahashi T, Matoba S, Yamada H, Okigaki M and Matsubara H: Replicative senescence of vascular smooth muscle cells enhances the calcification through initiating the osteoblastic transition. Am J Physiol Heart Circ Physiol 297: H1673-H1684, 2009.

12. Iijima K: Bone and calcium update; diagnosis and therapy of bone metabolism disease update. Regulatory mechanism of mammalian sirtuin SIRT1 in vascular calcification: Impact of vascular smooth muscle cell senescence. Clin Calcium 21: 53-60, 2011 (In Japanese).

13. Zhan JK, Wang YJ, Wang Y, Tang ZY, Tan P, Huang W and Liu YS: Adiponectin attenuates the osteoblastic differentiation of vascular smooth muscle cells through the AMPK/mTOR pathway. Exp Cell Res 323: 352-358, 2014.

14. Tan P, Wang YJ, Li S, Wang Y, He JY, Chen YY, Deng HQ, Huang W, Zhan JK and Liu YS: The PI3K/Akt/mTOR pathway regulates the replicative senescence of human VSMCs. Mol Cell Biochem 422: 1-10, 2016.

15. Zhan JK, Wang YJ, Wang Y, Tang ZY, Tan P, Huang W and Liu YS: The protective effect of GLP-1 analogue in arterial calcification through attenuating osteoblastic differentiation of human VSMCs. Int J Cardiol 189: 188-193, 2015

16. Inoki K, Kim J and Guan KL: AMPK and mTOR in cellular energy homeostasis and drug targets. Annu Rev Pharmacol Toxicol 52: 381-400, 2012. 